\title{
3D Printed Porous Media Columns with Fine Control of Column Packing Geometry
}

\author{
Conan Fee*, Suhas Nawada, Simone Dimartino
}

Department of Chemical \& Process Engineering and Biomolecular Interaction Centre, University of Canterbury, Private Bag 4800, Christchurch 8020, New Zealand

*Corresponding Author:

Email: conan.fee@canterbury.ac.nz

Phone: +64 33642139

Fax: +64 3642063

(1)

\section{Abstract}

In this paper we demonstrate, for the first time, the use of 3D printing (also known as additive manufacturing or rapid prototyping) to create porous media with precisely defined packing geometries, directly from computer aided design (CAD) models. We used CAD to design perfectly ordered beds with octahedral beads (115 $\mu \mathrm{m}$ apothem) packed in a simple cubic configuration and monoliths with hexagonal channels (150 $\mu \mathrm{m}$ apothem) in parallel and herringbone arrangements. The models were then printed by UV curing of acrylonitrile-butadiene-styrene powder layers. Each porous bed was printed at 1.0, 1.5 and $2.0 \mathrm{~mL}$ volumes, within a complete column, including internal 6 flow distributors and threaded 10-32 flow connectors. Close replication of CAD 7 models was achieved. The resultant individual octahedral beads were highly uniform in size, with apothems of $113.6 \pm 1.9 \mu \mathrm{m}$, while the monolith hexagonal cross-section 9 channels had apothems of $148.2 \pm 2.0 \mu \mathrm{m}$. Residence time distribution measurements show that the beds largely behaved as expected from their design void volumes.

1 Radial and fractal flow distributor designs were also tested. The former displayed 2 poor flow distribution in parallel and herringbone pore columns, while the fractal 3 distributors provided uniform flow distribution over the entire cross section. The 
results show that $3 \mathrm{D}$ printing is a feasible method for producing precisely controlled porous media. We expect our approach to revolutionize not only fundamental studies of flow in porous media but methods of chromatography column production.

Keywords: Porous media; 3D printing; Additive manufacturing; Packed bed; Packing geometry; Residence time distribution

\section{Introduction}

Porous media are important for fluid-solid contacting in many unit operations, including adsorption, chromatography, catalysis and filtration. Media particles are typically packed into a column, allowing fluid to flow through the interstitial voids, thus bringing the fluid into close contact with the solid phase. Key to the effectiveness of packed columns are the flow-related properties of mass transfer, fluid distribution, back pressure and fluid dispersion, which in turn depend upon packing geometry.

Packing geometry is determined primarily by particle shape, size and size distribution and the packing method used. While there have been many theoretical studies on optimal packing configurations and their effects on packing density, along with computational studies on theoretical plate height and flow dispersion (e.g. [1]), packed beds have, for practical reasons, invariably been randomly packed to date. Thus, there has been no way before now to translate optimal ordered packing arrangements into practice.

There have been many studies on flow through randomly packed beds, notably the seminal works of Darcy [2], Kozeny [3], Carman [4] and Ergun [5]. These and other authors have contributed much to our understanding of pressure drop and fluid dispersion as functions of flow rate, particle shape, size and size distribution, largely based upon empirical characterization. Experimental replication of models with specific random or ordered packing geometries has been challenging. For random geometries, no two randomly packed beds are exactly alike so we rely upon generalized correlations and efficiency factors where, to quote Khirevich et al. [6]: "column packing and consolidation are largely treated phenomenologically and 
considered an art rather than a science". On the other hand, it has been impracticable to precisely reproduce ordered packing at the micron scale, mainly because there has been no practical way to precisely locate individual particles within a bed. Even if precise placement of the particles were feasible, e.g. through manual placement of each bead, the column walls would almost certainly frustrate attempts to maintain order.

Some authors (e.g. [7-10]) have characterized existing randomly packed beds through tomography, thus reproducing, a posteriori, the geometry of their experimental columns for computational analysis. However, they have had no control over the initial packing of the experimental beds at the individual particle level so it has been a case of accepting, rather than a priori designing, the fine structural detail.

Furthermore, because individual particles may change their positions with time, the characterization of packing geometries is valid only as a snapshot in time.

Efforts to optimize the performance of packed beds for chromatography have focused on the manufacture of bed particles (resin) and, because of ease of manufacture and guaranteed bed permeability, these have been predominantly spherical [11]. Many methods have been developed for producing spherical beads in bulk but they typically result in wide particle size distributions, which are minimized in final media products by fractionation, leading to increased costs, inefficient production and ultimately variations in packing geometry through size variation in all but the most expensive of media.

In this paper, we introduce an entirely new approach to packed column manufacture that solves many of the above problems, using 3D printing (also known as "additive manufacturing" or "rapid prototyping") to produce packed beds that precisely replicate computer aided design (CAD) models. 3D printing is a generic term for techniques by which solid objects are created from digital models. The first working 3D printer was patented by Hull with a priority date of 1984 [12]. Since then, a variety of 3D printing systems have been developed, including fused deposition, selective heat or laser sintering, photopolymerization and thin-film lamination. Several recent reviews of the development and advances in 3D printing are available [13-17]. 
103 We use the term "packed" above advisedly because our technique produces what

104 might better be described as monoliths, although, as described below, they are distinct

105 from monoliths in their current sense in chromatography, which effectively exchange

106 random particle packing for random pore geometries [18, 19]. In contrast, with our

107 approach, we have created and tested exact physical replicas of ordered packed bed

108 CAD models, comprising ordered arrays of uniform particles. Our approach opens up

109 the possibility, for the first time, to precisely locate and orient every individual

110 particle within a porous bed. Here, we also demonstrate the production of monoliths

111 with precise internal pore geometries and, moreover, show that we can print not only

112 the porous bed but the entire column, complete with internal flow distributors,

113 packing, and external fluid connectors, therefore creating single-piece

114 chromatography columns.

\section{Materials and Methods}

117 Stereolithography (STL) files for the column models were created on Solidworks

1182012 (Dassault Systèmes, Paris, France) and printed on a 3DS Projet HD 3500 printer

119 (3D Systems, Rock Hill, SC, USA). The printed components were made from non-

120 porous urethane acrylate oligomers (acrylonitrile butadiene styrene, ABS). A

121 proprietary paraffin wax was also used by the $3 \mathrm{D}$ printer during printing to support

122 overhanging features. The wax was removed from the internal structures of the

123 columns by alternating warm water $\left(70^{\circ} \mathrm{C}\right)$ and $100 \%$ cyclohexane washes for up to 3

$124 \mathrm{~h}$.

126 The CAD models included the "packed" porous core and the ancillary column

127 elements, namely column walls, fluid distributors and collectors and end fittings for

128 easy connection to the experimental chromatography system. This enabled our

129 "packed" columns to be printed as an all-in-one parts, with no further assembly

130 required before use.

The porous beds were created with three geometries: beads in a simple cubic

135 arrangement (SC, Fig. 1a), a monolith containing parallel channels (PC, Fig. 1b) and

136 another containing herringbone shaped channels (HC, Fig. 1c). 
137 The nominal resolution of the 3D printer was $28 \mu \mathrm{m}$, but the limiting dimensions of

138 the lattice elements that could be reliably printed at the desired resolution were about

139 one order of magnitude larger. Polyhedrons and polygons were used to design the

140 "packing" elements rather than spheres and circles, to minimize the file size of the

141 STL models while maintaining a regular shape in the lattice elements. For this reason,

142 octahedral beads (115 $\mu \mathrm{m}$ apothem) were used in the SC arrangement, while channels

143 with hexagonal cross-sections (150 $\mu \mathrm{m}$ apothem) were used for both the PC and HC

144 configurations. The HC geometry was designed with a tortuosity of 1.15 , where the

145 tortuosity is defined as the ratio between the total length of the channels and the

146 column height.

147

148

149

150

151

152

153

154

155

156

157 Columns with total bed volumes of $1,1.5$ and $2 \mathrm{ml}$ were produced for each packed

158 bed geometry studied. The internal diameter and wall thickness of the cylindrical

159 columns were 16 and $2 \mathrm{~mm}$, respectively. Connection with the chromatography

160 system was facilitated by including a 10-32 standard coned, female, fast protein liquid

161 chromatography (FPLC) finger-tight fitting at each end of the columns. All columns

162 contained a flow distributor at the porous bed entrance and an identical flow collector

163 at the outlet. Two geometric designs for the flow distributor and collector were used, a

164 standard radial distributor comprising a set of concentric and radial channels, and a

165 fractal flow distributor with square cross-section and 1024 nodes as proposed by

166 Tondeur and Luo [20]. The corresponding printed columns, therefore, were of circular

167 and square cross-sections, accordingly to the flow distributor considered. Figure 2

168 presents solid models of the flow channels within each distributor (collector) design,

169 which were then subtracted from the solid ends of the columns in the CAD model to 
170 produce the flow channels. The characteristics of the 3D printed columns are

171 summarized in Table 1.

172

173 Residence time distribution (RTD) tests were carried out using an ÄKTA explorer

$17410^{\mathrm{TM}}$ FPLC system equipped with an auto-sampler (GE Healthcare, Uppsala,

175 Sweden). The columns were first equilibrated with pure water for 15 column volumes

$176(\mathrm{CV})$, followed by injection of $30 \mu \mathrm{l}$ of $2 \mathrm{M} \mathrm{NaCl}$. RTD experiments were carried out

177 at a flow rate of $10 \mathrm{ml} / \mathrm{min}$, which corresponds to superficial velocities of 298 and 295

$178 \mathrm{~cm} / \mathrm{h}$ for the circular and square cross-section columns, respectively. The conductivity

179 peak in the column effluent was recorded and analyzed using the moment method.

180 The injected volume was $6 \%$ or less of the void volumes of the columns tested, hence

181 the contribution to the first moment arising from the injection loop can be neglected.

182 Under this assumption, the experimental residence time, $t_{r}^{\text {exp }}$, can be calculated as:

$t_{r}^{\exp }=\frac{M_{1}}{M_{0}}=\frac{\int_{0}^{\infty} c(t) t d t}{\int_{0}^{\infty} c(t) d t}$

184 where $M_{\mathrm{i}}$ is the $\mathrm{i}^{\text {th }}$ absolute moment, $c$ is the concentration of the tracer, and $t$ is time.

185 E curves, i.e. normalized elution profiles having unitary area, were calculated from

186 the conductivity signal and the $0^{\text {th }}$ moment:

$187 E(\theta)=\frac{c(\theta)}{M_{0}}$

188 where $\theta$ is the dimensionless time defined in terms of the theoretical residence time,

$189 t_{r}^{\text {theo }}$, estimated from the designed geometry of the lattice structure:

$190 \quad \theta=\frac{t}{t_{r}^{\text {theo }}}$

191 This expression can be also used to define an experimental dimensionless residence

192 time, $\theta_{r}^{\exp }$ :

$193 \quad \theta_{r}^{\exp }=\frac{t_{r}^{\exp }}{t_{r}^{\text {theo }}}$

194 Comparison of the theoretical and experimental residence times was used to assess the

195 quality of the printed lattices and the uniformity of the flow distribution. 


\section{Results and Discussion}

199 Creation of STL models represents the first step in the production of the 3D printed

200 porous columns. Definition of the building elements of the lattice is crucial, in part

201 because this is the attribute that has the most influence on file size and subsequent file

202 handling. Contribution to the final file size arising from column walls, end fittings,

203 and distributor/collector can be neglected. In the initial design, spherical beads and

204 circular channels were considered but the STL file sizes were too large, from both the

205 viewpoints of the speed of rendering during CAD modeling and of the printer file

206 handling capacity. For example, to accurately model a single sphere, irrespective of

207 diameter, our CAD package used approximately 6162 triangles, with a file size of 306

208 kbytes, while an octahedron was described by only 8 triangles, giving a file size of

209 less than 0.5 kbytes, three orders of magnitude smaller. However, file size per se is

210 not fundamentally a limiting factor for 3D printing, and could be handled with

211 efficient computational algorithms or compression, particularly with the constantly

212 growing capacity of microprocessors, communications and storage media with time.

213 Furthermore, ordered packing geometries are based on repetitive structures that would

214 lend themselves to iterative printer command sequences. Thus, there is no

215 fundamental reason why spherical elements could not be used, given sufficient

216 software and hardware processing power.

218 It is well understood that in 2D image processing, the resolution of a picture is

219 proportional to the number of pixels of which it is comprised. Similarly, the quality of

220 the rendering of a solid shape is proportional to the number of $3 \mathrm{D}$ dots used to

221 discretize it. The resolution of a 3D printer is an indication of the size of the smallest

222 feature that is possible to print, i.e. it corresponds to the dimensions of the "3D dots"

223 that make up the printed model. However, the final size and shape of the 3D dots are

224 ultimately determined by a number of uncontrollable variables. In our case, using

225 layer deposition followed by UV curing, examples of these uncontrollable variables

226 are: i) the interfacial forces acting between the ABS polymer and the support material,

227 ii) the local temperature of the ABS polymer during UV curing, iii) venting

228 characteristics of the printing chamber, iv) defects and irregularities during layering of

229 the ABS powder. 
231 Also, the resolution quoted by a 3D printer manufacturer may well comprise the best 232 possible that can be achieved under ideal conditions but this may not be routinely

233 achievable in normal practice. The 3D printer used in this work had a nominal

234 resolution of $28 \mu \mathrm{m}$ so the printed octahedral particles or hexagonal cross-section

235 channels were characterized by relatively rounded edges at the micron scale.

236 However, as is shown in the following discussion, the features of the CAD models

237 were conserved in the 3D printed objects, hence microscopic limitations in the

238 resolution do not represent a significant limitation of 3D printed porous media.

240 In addition to full operational columns, cross-sectional "cutaway" models of each

241 packing configuration were printed to display the internal structures of the columns,

242 distributors and porous beds. Figure 3 shows that not only the column macrostructures

243 but also the microstructures of the CAD models were reproduced with reasonable

244 fidelity by the printer.

246 Inspection of Fig. 3 reveals that the mean bead and channel apothems were $113.6 \pm$ 2473.8 and $148.2 \pm 2.0 \mu \mathrm{m}$, respectively, while the design values were 115 and $150 \mu \mathrm{m}$, 248 respectively, demonstrating the precise control over packed bed microstructures 249 delivered by our 3D printing approach. Figure 3c shows a magnification of the simple

250 cubic cutaway model, showing that the particles were approximately octahedral and 251 the dimensions of the pores and relative diameters of the beads were consistent with 252 the design compression factor $\alpha=1.4$. Likewise, magnified images of the straight and 253 herringbone channels (Fig. 3f and 31) show reasonable fidelity between the CAD 254 models and the printed columns in the cutaway models, revealing that the 3D printer 255 used was able to reproduce the CAD models well. It is reasonable to expect the same 256 fidelity was obtained between the CAD models and the full operational printed 257 columns.

259 Residence time distribution (RTD) tests were conducted on all 3D printed columns, 260 first, to highlight differences between the "packing" geometries used and, second, to 261 compare the effectiveness of the two distributor designs. 
263 Normalized residence time distribution profiles of the printed columns are shown in

264 Fig. 4. We were concerned that the printer might not faithfully reproduce octahedral

265 beads but rather create roughly spherical beads because of limitations in resolution.

266 However, evidence of the high fidelity between the CAD model and the 3D printed

267 columns can be found in the RTD experiments by comparing theoretical and

268 experimental porosity values. At $\alpha=1.4$, the designed extra-particle porosity of the

269 simple cubic octahedral beads is $\varepsilon=0.575$. For comparison, a simple cubic

270 configuration of spherical beads with the same overlap would have a theoretical

271 porosity of $\varepsilon=0.041$, while it would be $\varepsilon=0.476$ with no overlap. The

272 experimentally determined porosities of $\varepsilon=0.678,0.569$ and 0.551 for $1,1.5$ and $2 \mathrm{ml}$

273 columns are closer to the design porosity for octahedral beads $(17.9 \%, 1.0 \%$ and $4.2 \%$

274 differences, respectively) rather than that for spherical particles (minimum difference

$27534.4 \%$ ), suggesting good control over particle shape at the 3D printer's limiting

276 resolution. The mean normalized residence times for simple cubic bead columns also

277 indicate reasonable consistency between the design (expected) and experimental

278 column porosities.

280 The low mean residence times in the straight and herringbone channel cylindrical

281 columns (Fig. 4a) compared with the cubic packing suggest that a substantial

282 proportion of the channels in those columns were not accessed by the fluid when

283 using radial flow distributors. This is a strong indication of the low efficiency of the

284 radial distributor, which was not able to spread the incoming flow uniformly over the

285 entire cross section. The radial distributor primarily conveys the flow through the

286 central channel, while there is no reason for flow to move radially in the distributor

287 unless there is an axial flow resistance in the bed. In the case of cubic packing, the

288 interconnected network of beads allows for both radial and axial dispersion, so it is

289 the packing itself that assists in the uniform distribution of the flow across the cross-

290 section, giving an experimental dimensionless residence time, $\theta_{r}^{\text {exp }}$, close to unity

291 (Fig. 4 and Table 2). In contrast, because radial dispersion is absent throughout the

292 parallel and herringbone channel columns, the fluid would have followed only the

293 channels into which it initially entered. It is likely, therefore that the performance of

294 these latter columns was limited by inadequate radial flow distribution at entry,

295 resulting in $\theta_{r}^{\text {exp }}<1.0$ in the RTDs shown in Figure 4a. 
297 In an attempt to improve uniform flow distribution over the entire cross-section, a

298 fractal distributor was designed. For both the radial (circular column cross-section)

299 and fractal (square column cross-section) designs, we printed shortened columns

300 containing no bed but with the inlet flow distributor and outlet flow collector placed

301 immediately adjacent to one another and compared their residence time distributions.

302 This approach may also be useful to measure extra-bed dispersion but in this case we

303 simply compared the average residence times of the two distributor designs. The

304 mean residence times for the radial and fractal distributors were $\theta_{r}^{\exp }=0.32$ and 0.93 ,

305 respectively, indicating that flow was not well distributed in the radial flow

306 distributor, while it was relatively uniformly distributed in the fractal design.

308 Figure 5 compares the normalized RTD curves in $2 \mathrm{ml} \mathrm{PC} \mathrm{(Fig.} \mathrm{5a)} \mathrm{and} \mathrm{SC} \mathrm{(Fig.} \mathrm{5b)}$

309 columns containing the two distributor designs. Note that there is a significant

310 difference between the mean elution times for the two distributor designs in the PC

311 column, in which there was no radial dispersion within the bed itself, while in the

312 simple cubic bead column, where the bed itself provides radial distribution, there was

313 little difference between the RTDs for the two distributor designs. Thus, for bed pore

314 geometries that do not promote radial flow dispersion, careful design of the fluid

315 distributor is required.

317 Note also, in Fig. 5b, that the fractal distributor system for the SC column resulted in

318 a longer tailing in the RTD curve. This possibly occurs because the flow rate in the

319 corners of the square cross-section column with the fractal distributor may be slower

320 for the SC packing than the mean flow rates in the rest of the bed, leading to greater

321 axial dispersion than that in the cylindrical cross-section column. These differences in

322 tailing are not seen between the two PC columns (Fig. 5a) because the axial

323 dispersion is affected only by the flow through the independent channels, which have

324 uniform geometry, regardless of the overall column cross-sectional geometry.

326 Pressure-flow measurements of the printed columns were found to be

327 indistinguishable from control measurements using just the FPLC system in by-pass

328 mode (i.e. with no column attached, data not shown). This result is consistent with 
329 expectations because short columns were used, resulting in low column

330 backpressures. No external structural defects nor leaks were observed at superficial

331 velocities of up to $594 \mathrm{~cm} \cdot \mathrm{hr}^{-1}$ (corresponding to $20 \mathrm{ml} \cdot \mathrm{min}^{-1}$ ), demonstrating the

332 structural robustness of the all-in-one-piece 3D printed columns.

334 Our choice of materials for this work was constrained to those that were readily

335 available for rapid prototyping, so we used a non-porous material and focused on

336 demonstrating that we could achieve control over packing geometry, at least within

337 the resolution of the particular printer used here. Clearly, an ideal chromatographic

338 media would comprise finer-resolution elements to minimize the theoretical plate

339 height, a functionalizable surface chemistry to enable ligand attachment for reversible

340 adsorption, and porous materials to maximize adsorption capacity. We have not

341 demonstrated these ideal characteristics in this paper but see no fundamental reasons

342 why all of these ideal characteristics could not be achieved. We also limited the

343 volumes of our columns to those that were convenient to handle in the laboratory.

344 However, even with the printer used here, there is no particular reason why we could

345 not have produced columns with significantly greater dimensions, at the same fine

346 resolution but with a diameter and length of $30 \mathrm{~cm}$ or more i.e. preparative scale. The

347 materials used here were low-cost and in general, the use of materials in 3D printing

348 is very efficient, using only the amount necessary to produce the specific features of

349 the CAD model. Thus, we believe our approach is scalable and will enable not only

350 fundamental studies of flow, mass transfer and adsorption through structured porous

351 media but perhaps, in time, commercial column production.

353 Our approach can be applied not only to chromatography but to any application

354 requiring fluid-solid contacting, including filtration and catalytic or other reaction

355 applications. One could create precise replicates of randomly packed beds, to enable

356 experimental validation of computational models. Furthermore, the ability to orientate

357 the individual particles means we can go beyond using spheres and conceive beds

358 comprising unusual and complex particle shapes, while maintaining uniform porosity

359 throughout. There is no particular need for all elements within the bed to be uniform

360 with regard to size or shape and one could imagine porous media with a wide range of

361 controlled geometry elements throughout the bed could be designed and printed.

362 There are currently printers on the market capable of printing multiple materials at 
363 once (rather like a color inkjet printer) so the various column and bed components 364 could, in principle, be printed using different materials, each suited to its particular 365 purpose e.g. porous, functional bed materials with non-porous, inert column walls, 366 flow connectors and distributors.

368 Thus, there is enormous potential for using additive manufacturing to produce

369 versatile monolithic porous media with designed geometries not only for the beds 370 themselves but for the entire columns.

\section{Conclusions}

374 We have shown, for the first time, that 3D printing can be used to precisely replicate 375 the fine structure of CAD models of porous media, comprising both ordered particle 376 packing and monoliths with internal channels. Residence time distributions measured 377 in the printed columns were consistent with predicted porosities and designed 378 geometric structures, indicating that the CAD features were reproduced with good 379 fidelity at the scales attempted here.

381 Furthermore, we have demonstrated that not only the porous beds themselves but 382 entire columns can be printed as single physical artifacts, meaning that flow 383 connectors, flow distributors and internal column packing can be printed within a 384 single, complete column. Fractal flow distributors are capable of distributing the flow 385 across the entire column cross-section, even when there is no radial dispersion across 386 the flow channels of monolithic beds. This distributor design enabled the creation of 387 square cross-section monolithic columns with good flow distribution and residence time distributions through parallel channels that were independent of the column

$3913 \mathrm{D}$ printing frees us from the constraints of previous manufacturing methods and 392 enables the creation of porous media characterized by a combination of fine precision, 393 scalability and versatility, at low cost. We expect this approach to column design will 394 revolutionize the production of packed bed columns and monoliths across a wide 395 range of applications, not only in chromatography but also filtration, catalysis, 396 adsorption and other applications where intimate fluid-solid contact is desired. 


\section{Acknowledgement}

399 S. Nawada gratefully acknowledges a PhD scholarship from the Biomolecular

400 Interaction Centre, University of Canterbury. This work was partially funded by a

401 Ministry of Business, Innovation and Employment Smart Ideas Grant UOCX1304.

402

403

404

\section{References}

405

1. M.R. Schure, R.S. Maier, J. Chrom. A 1126(1-2) (2006) 58.

406 2. H. Darcy, Les Fontaines Publiques de la Vile de Dijon, Victor Dalmont, Paris, $407 \quad 1856$.

408 3. J. Kozeny, Sitzungsber Akad. Wiss 136(2a) (1927) 271.

4094 4.C. Carman, Trans. Inst. Chem. Eng. Lond. 15 (1937) 150.

410 5. S. Ergun, Chem. Eng. Prog. 48(2) (1952) 89.

411 6. S. Khirevich, A. Daneyko, A. Höltzel, A. Seidel-Morgenstern, U. Tallarek, J. $412 \quad$ Chrom. A 1217(28) (2010) 4713.

413 7. A. Akolkar, J. Petrasch, Transp. Porous Media 95(3) (2012) 535.

414 8. Y.X. Cao, B. Chakrabortty, G.C. Barker, A. Mehta, Y.J.Wang, Europhys. 415 Lett. 102(2) (2013) 24004-p1.

416 9. R. Moreno-Atanasio, R.A. Williams, X.D. Jia, Particuology 8(2) (2010) 81.

417 10. M. Schubert, A. Bieberle, F. Barthel, S. Boden, U. Hampel, Chem. Ing. Tech. 418 83(7) (2011) 979.

419 11. M.T. Gokmen, F.E. Du Prez, Prog. Polym. Sci. 37(3) (2012) 365.

420 12. C. W. Hull, US Patent 4575330 (1986).

421 13. D.H. Freedman, Technol. Rev. 115(1) (2012) 50.

422 14. R.D. Goodridge, C.J. Tuck, R.J.M. Hague, Progr. Mat. Sci. 57(2) (2012) 229.

423 15. D.D. Gu, W. Meiners, K. Wissenbach, R. Porawe, Int. Mat. Rev. 57(3) (2012) $424 \quad 133$.

425 16. S. Kumar, J.P. Kruth, Mater. Des. 31(2) (2010) 850.

426 17. Wendel, B., et al., Additive Processing of Polymers. Macromolecular 427 Materials and Engineering, 2008. 293(10): p. 799-809.

428 18. A. Nordborg, E.F. Hilder, Anal. Bioanal. Chem. 394(1) (2009) 71.

429 19. Z. Walsh, B. Paull, M. Macka, Anal. Chim. Acta 750 (2012) 28.

430 20. D. Tondeur, L.G. Luo, Chem. Eng. Sci. 59(8-9) (2004) 1799. 
438 Figure 2. Illustration of the flow distributor templates: a) radial flow distributor, b)

439 fractal flow distributor (Note: for clarity, only the first 64 of the 1024 nodes are 440 shown)

441

\section{Figure Legends}

Figure 1. The three bed geometric designs: a) simple cubic beads, b) straight channels, c) herringbone channels

Figure 3. CAD designs versus printed cutaway columns a) SC CAD model b) SC printed model c) 20X magnification of SC beads d) PC CAD model e) PC printed model f) 20X magnification of parallel channels g) HC CAD model h) HC printed model 1) 20X magnification of herringbone channels

Figure 4. Residence time distribution profiles of a) the three packing geometries in $1.5 \mathrm{ml}$ cylindrical columns, b) SC bead columns at three different column volumes.

Figure 5. Comparison of $2 \mathrm{ml}$ columns with radial and fractal flow distributors for a) PC columns b) SC columns 
Table 1. Specifications of 3D Columns

\begin{tabular}{ccccc}
\hline $\begin{array}{c}\text { Column } \\
\text { Cross- } \\
\text { Section }\end{array}$ & $\begin{array}{c}\text { Flow } \\
\text { Distributor }\end{array}$ & $\begin{array}{c}\text { Column } \\
\text { Volumes } \\
(\mathbf{m l})\end{array}$ & $\begin{array}{c}\text { Packing } \\
\text { Configuration }\end{array}$ & $\begin{array}{c}\text { Theoretical } \\
\text { Porosity }\end{array}$ \\
\hline Circular & Radial & $1.0,1.5,2.0$ & SC & 0.575 \\
Circular & Radial & $1.0,1.5,2.0$ & PC & 0.334 \\
Circular & Radial & $1.0,1.5,2.0$ & HC & 0.334 \\
Square & Fractal & 2.0 & SC & 0.575 \\
Square & Fractal & 2.0 & PC & 0.393 \\
\hline
\end{tabular}


Table 2. Theoretical and experimental mean residence times of SC columns

\begin{tabular}{cccc}
\hline $\begin{array}{c}\text { Column Volume } \\
(\mathbf{m l})\end{array}$ & $\begin{array}{c}\boldsymbol{t}_{\boldsymbol{r}}^{\text {theo }} \\
(\mathbf{m i n})\end{array}$ & $\begin{array}{c}\boldsymbol{t}_{\boldsymbol{r}}^{\exp } \\
(\mathbf{m i n})\end{array}$ & $\begin{array}{c}\boldsymbol{\theta}_{\boldsymbol{r}}^{\text {exp }} \\
(-)\end{array}$ \\
\hline 1.0 & 0.705 & 0.804 & 1.14 \\
1.5 & 0.993 & 0.993 & 1.00 \\
2.0 & 1.280 & 1.229 & 0.96 \\
\hline
\end{tabular}


Figure 1

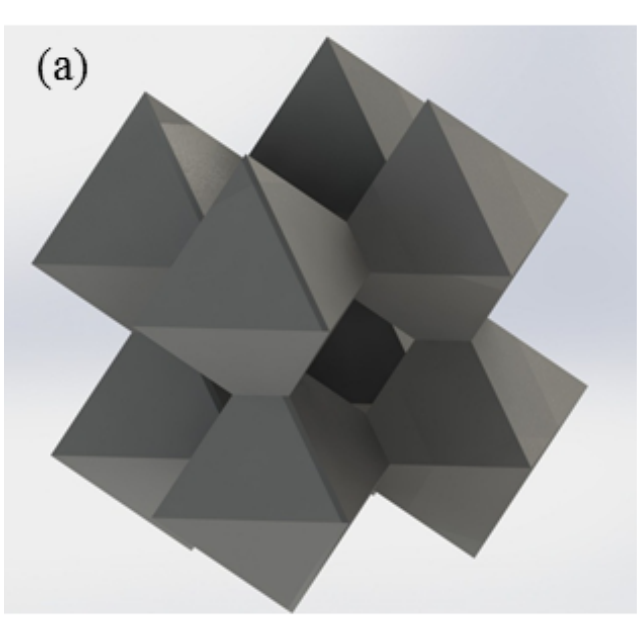

(b)

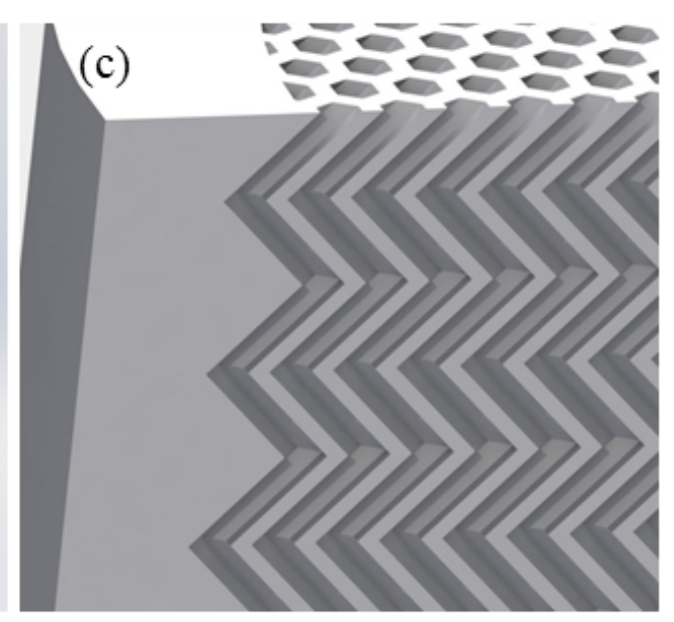


Figure 2

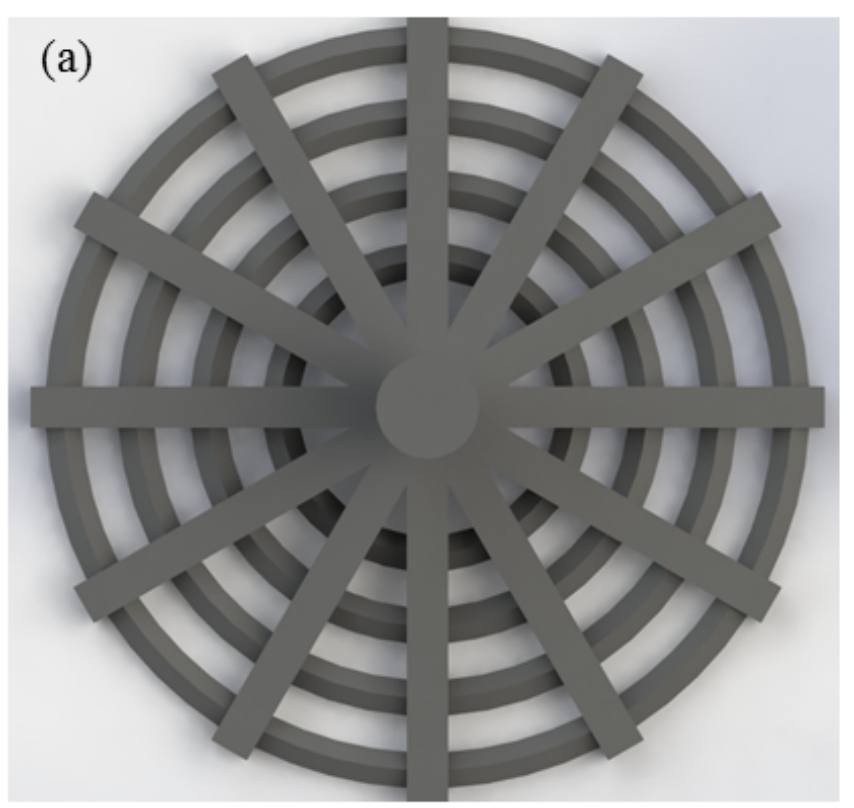

(b)

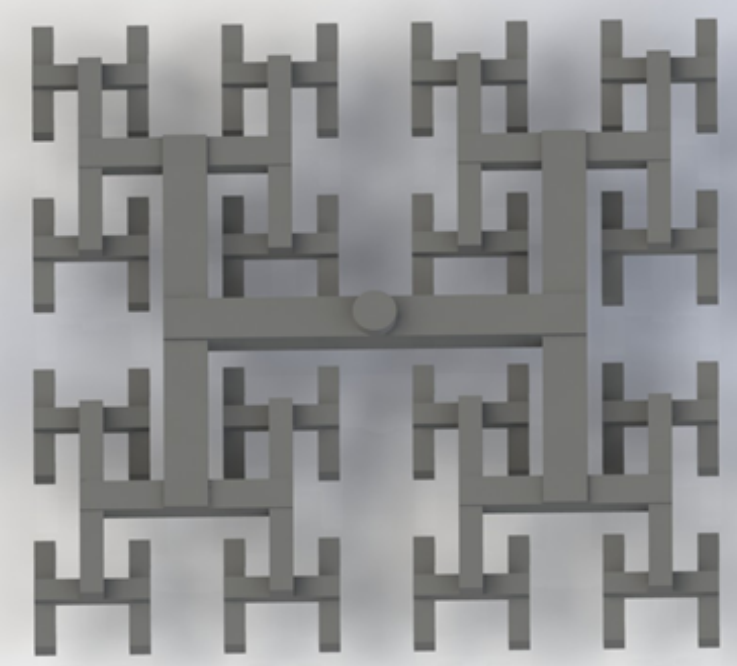


Figure 3
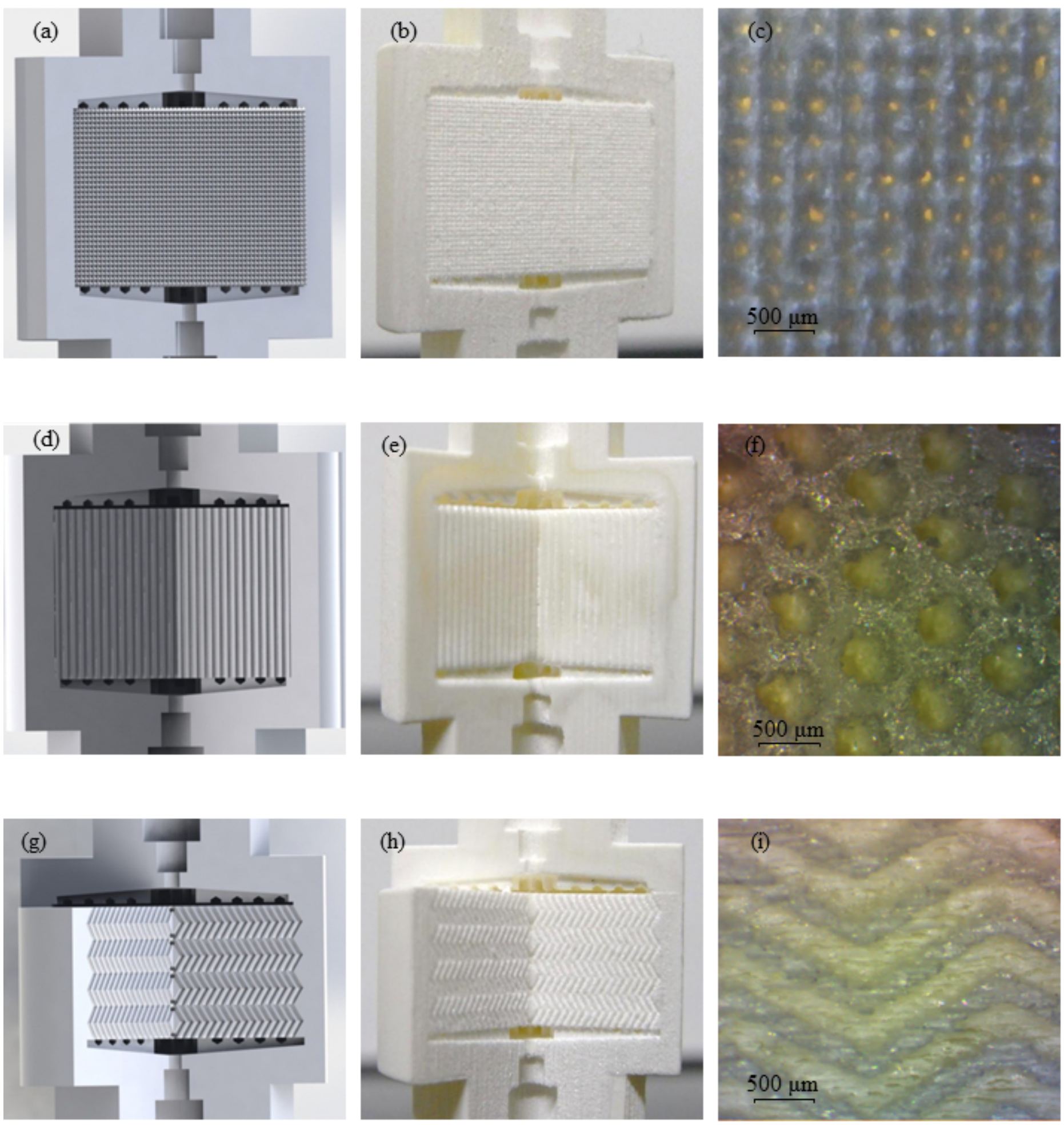
Figure 4
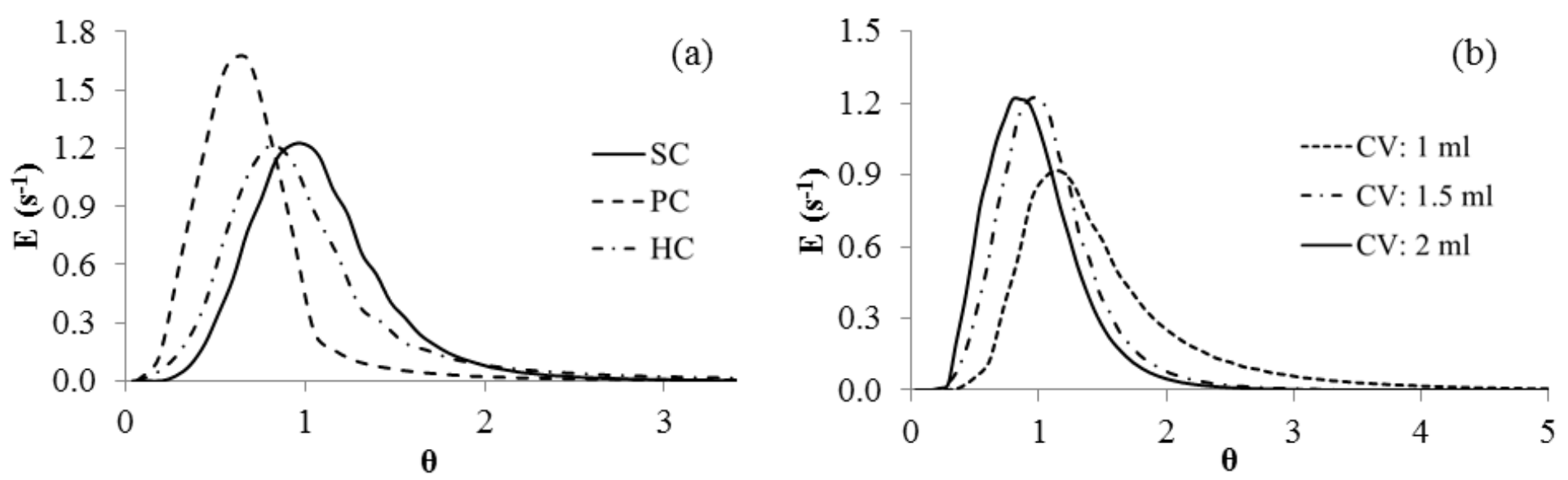
Figure 5
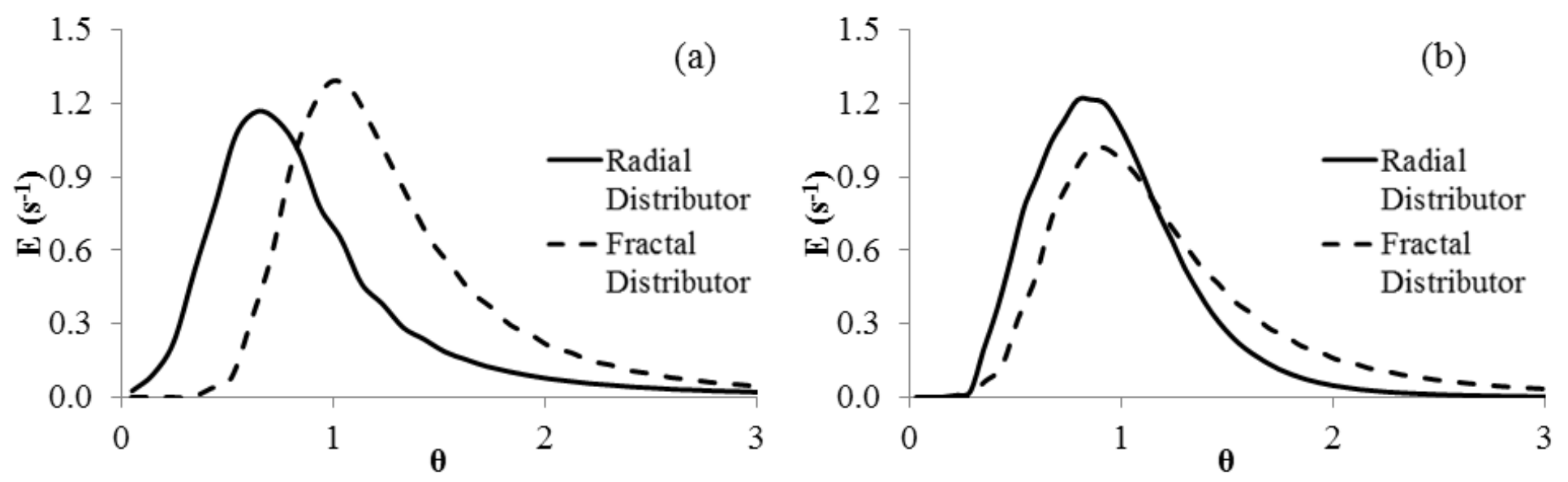